7 Crawford J, Armitage J, Balducci L et al. Myeloid growth factors. J Natl Compr Canc Netw 2013;11:1266-90.

8 Royal College of Physicians. Acute care toolkit 7. Acute oncology on the acute medical unit. London: RCP, 2013. www.rcplondon.ac.uk/sites/ default/files/acute_care_toolkit_7.pdf [Accessed 5 August 2014].

9 NHS National Cancer Action Team. National Cancer Peer Review Programme. Manual for cancer services: acute oncology - including metatastic spinal cord compression measures (Version 1.0). London: National Cancer Action Team, 2011. www.gov.uk/government/ uploads/system/uploads/attachment_data/file/216121/dh_125889.pdf [Accessed 5 August 2014].

10 Klastersky J, Paesmans M, Edward EB et al. The Multinational Association for Supportive Care in Cancer risk index: a multinational scoring system for identifying low risk febrile neutropenic cancer patients. J Clin Oncol 2000;18:3038-51.
11 Innes H, Lim S, Hall A et al. Management of febrile neutropenia in solid tumours and lymphomas using the Multinational Association for Supportive Care in Cancer (MASCC) risk index: feasibility and safety in routine clinical practice. Support Care Cancer 2008;16:485-91.

12 National Chemotherapy Advisory Group. Chemotherapy services in England: ensuring quality and safety. London: National Chemotherapy Advisory Group, 2009. http://webarchive.nationalarchives.gov.uk/ 20130107105354/http://www.dh.gov.uk/prod_consum_dh/groups/ dh_digitalassets/documents/digitalasset/dh_104501.pdf [Accessed 5 August 2014].

Address for correspondence: Dr E Marshall,

Clatterbridge Cancer Centre, Clatterbridge Road, Bebington, Wirral CH63 4JY.

Email: emarshall@nhs.net

\title{
Metastatic spinal cord compression: a rare but important complication of cancer
}

\author{
Author: Peter Robson ${ }^{A}$
}

\section{Background}

Metastatic spinal cord compression (MSCC) is a well-recognised complication of cancer and usually presents as an oncological emergency. Metastases to the spine occur in 3-5\% of all patients who have cancer and are more common in patients with breast, prostate and lung cancer, in whom the incidence is $19 \% .^{1}$ The true incidence of MSCC is unknown but estimates are around $15 \%$ of patients with advanced cancer. ${ }^{2}$

MSCC is usually caused by the collapse or compression of a vertebral body that contains metastatic disease, but can also rarely be caused by direct tumour extension into the vertebral column. Compression of the cord initially causes oedema, venous congestion and demyelination, which are reversible. Prolonged compression leads to vascular injury, cord necrosis and permanent damage. Patients who have no neurological function for more than 48 hours are unlikely to improve. Their condition should be discussed with their primary tumour site clinician or oncologist before considering magnetic resonance imaging (MRI) or transfer. ${ }^{3}$

Author: ${ }^{A}$ consultant clinical oncologist, The Clatterbridge Cancer Centre NHS Foundation Trust, Bebington, UK

\section{Clinical symptoms and signs}

Back pain occurs in $95 \%$ of patients who present with MSCC. It is frequently the first symptom and often present for a prolonged period. The pain may present as spinal pain or radicular pain, and studies show that it is often of high intensity $(8 / 10)$. The next most common symptom of MSCC is limb weakness with many patients unable to walk unaided at presentation. ${ }^{3}$ Many patients who are at the point of developing MSCC are very unwell, have significant comorbidities or are of very poor performance status. Consideration should be made as to whether the patient would be fit enough for transfer and treatment before arranging an MRI. For a patient who is too frail or unfit for treatment, local palliative care is a more appropriate outcome; advice can be sought from the local acute oncology team or visiting oncologist.

Red flags for MSCC are: ${ }^{1}$

$>$ limb weakness

$>$ difficulty walking

$>$ sensory loss

$>$ bladder or bowel dysfunction

$>$ neurological signs

$>$ thoracic or cervical pain

$>$ pain that is increased by straining

$>$ nocturnal spinal pain. 


\section{NICE guidance}

National Institute for Health and Care Excellence (NICE) guidance CG75 (Nov 2008) has advocated the development of local cancer network pathways for the rapid diagnosis, treatment, rehabilitation and ongoing care of patients with MSCC. ${ }^{1}$ The following actions are recommended:

> patients who are at high risk of developing bone metastasis and those diagnosed with bony metastasis should be informed about the symptoms and signs of MSCC and given information informing them what to do if they develop these symptoms or signs

$>$ patients with spinal pain suggestive of metastasis and a diagnosis of cancer should have a whole-spine MRI within a week

> patients with signs of MSCC are a medical emergency and should have an urgent whole-spine MRI within 24 hours; ${ }^{4}$ you should:

- admit to hospital

- use bed rest with log-rolling

- administer dexamethasone $16 \mathrm{mg}$ daily with proton pump inhibitor (PPI) cover (unless there is a suspicion of lymphoma or another contraindication)

- provide adequate analgesia

> once MSCC is confirmed on MRI then contact the MSSC network coordinator for specialist input

> provide definitive treatment within 24 hours of confirmation of MSCC.

\section{Treatment options}

The definitive treatment options for a patient presenting with MSCC are surgery, radiotherapy or symptom control (Fig 1). Those patients who have had no motor function for over 48 hours are unlikely to recover any useful function following treatment. If this patient has significant pain, then treatment with a single fraction of 8 Gy radiotherapy should be considered for pain relief.

\section{NICE guidance}

National Institute for Health and Care Excellence (NICE) guidance CG75 (Nov 2008) has advocated the development of local cancer network pathways for the rapid diagnosis, treatment, rehabilitation and ongoing care of patients with MSCC. ${ }^{1}$ The following actions are recommended:

> patients who are at high risk of developing bone metastasis and those diagnosed with bony metastasis should be informed about the symptoms and signs of MSCC and given information informing them what to do if they develop these symptoms or signs

> patients with spinal pain suggestive of metastasis and a diagnosis of cancer should have a whole-spine MRI within a week

> patients with signs of MSCC are a medical emergency and should have an urgent whole-spine MRI within 24 hours; ${ }^{4}$ you should:

- admit to hospital

- use bed rest with log-rolling
Fig 1. Flow chart for diagnosis and treatment of metastatic spinal cord compression. $\mathrm{MRI}=$ magnetic resonance imaging; $\mathrm{MSCC}=$ metastatic spinal cord compression; $\mathrm{PPI}=$ proton pump inhibitor.
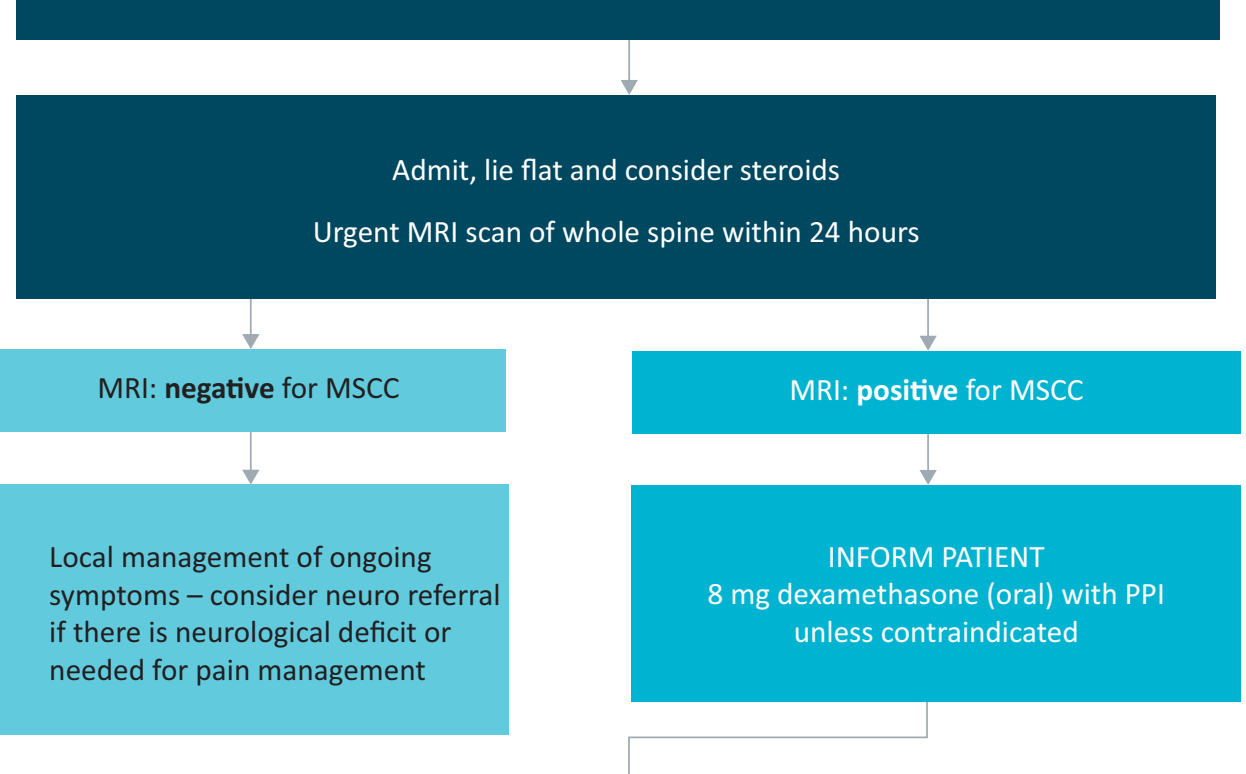

Contact MSCC coordinator to arrange senior review

Surgery followed by radiotherapy
Radiotherapy
Palliative care 


\section{Key points}

Metastatic spinal cord compression (MSCC) is an oncological emergency

All clinical staff and patients who are at high risk should be aware of the signs and symptoms of MSCC, and of what to do if they develop

Upon signs of MSCC, admit for bed rest, steroids and urgent magnetic resonance imaging (MRI) within 24 hours

Spinal pain that is suggestive of metastasis in a patient with cancer indicates a requirement for MRI within 1 week

There should be a network-led service for the delivery of services for MSCC patients

KEYWORDS: Metastatic spinal cord compression, radiotherapy, surgery, palliative

- administer dexamethasone $16 \mathrm{mg}$ daily with proton pump inhibitor (PPI) cover (unless there is a suspicion of lymphoma or another contraindication)

- provide adequate analgesia

> once MSCC is confirmed on MRI then contact the MSSC network coordinator for specialist input

$>$ provide definitive treatment within 24 hours of confirmation of MSCC.

\section{Treatment options}

The definitive treatment options for a patient presenting with MSCC are surgery, radiotherapy or symptom control (Fig 1). Those patients who have had no motor function for over 48 hours are unlikely to recover any useful function following treatment. If this patient has significant pain, then treatment with a single fraction of 8 Gy radiotherapy should be considered for pain relief. ${ }^{1}$

\section{Surgery}

For fit patients who have a good prognosis, decompressive surgery followed by radiotherapy is the treatment of choice. Surgery may also be considered to aid diagnosis by providing a biopsy or to stabilise an unstable spine in a patient with significant instability pain. Surgery may be the only effective option when there is compression of the cord by bony fragments following vertebral collapse. A computed tomographic (CT) scan can sometimes assist in this decision-making process. Staging CT scans are helpful in providing an impression of the extent of the patient's disease, but clinical judgment must be used in the context of a patient who has rapid neurological deterioration. Age is not a contraindication for surgery, but patients require careful selection as overall the elderly obtain relatively less benefit from surgery and radiotherapy. Major surgery should only be considered in those expected to live more than 3 months. ${ }^{1}$ Prior radiotherapy has been considered a contraindication for surgery, with wound breakdown or infection being three times more likely if radiotherapy has already taken place than if radiotherapy is performed after surgery. ${ }^{5}$

Prognostic indicators suggesting that surgery is more likely to be beneficial are: ${ }^{6}$

> histological findings such as multiple myeloma, lymphoma, or breast, prostate or renal cancers

$>$ good motor function at presentation

$>$ good performance status

$>$ limited comorbidity

$>$ single-level spinal disease

$>$ absence of visceral metastasis

$>$ long interval from primary diagnosis

The role of surgery has been addressed in a randomised controlled trial. ${ }^{7}$ Patchell et al looked at patients with single-level disease proven on MRI, good performance status and an onset of symptoms within 24 hours. They were randomised between circumferential decompressive surgery followed by radiotherapy (30 Gy in 10 fractions) or radiotherapy alone. Analysis showed a clear difference in the number of patients who were able to walk four steps post treatment: $84 \%$ of the combined (surgical) treatment group vs $57 \%$ of those treated with radiation alone $(\mathrm{p}=0.001)$. The surgical group were also able to walk for significantly longer (median 122 days vs 13 days, $\mathrm{p}=0.003$ ). In addition, the surgical group had higher rates of continence as well as better muscle strength and functional ability; they also required less opioid analgesics and corticosteroids. There is clear evidence that in this very select group of patients, surgery followed by radiotherapy is a beneficial treatment when compared with radiotherapy alone.

Nevertheless, the majority of patients presenting with MSCC have a poor prognosis, often with more extensive spinal disease and poor physiological reserve. The evidence to support surgery in this group is less clear, with studies showing only modest benefit for the addition of surgery to radiotherapy. ${ }^{8}$ Newer surgical techniques, involving percutaneous pedicle screws, cement augmented balloon kyphoplasty or a combination of the two, may be beneficial for this group of patients. ${ }^{9}$ Careful patient selection is paramount. Following surgery, all patients should be offered post-operative radiotherapy.

\section{Radiotherapy}

The majority of patients presenting with MSCC will be unsuitable for surgery. These patients should receive immediate radiotherapy (within 24 hours of MRI diagnosis of MSCC) as their definitive treatment. The aim of radiotherapy is to relieve compression of the spine and nerve roots by causing cell death in the rapidly dividing tumour tissue. This treatment is very effective at providing pain relief and is aimed at improving or stabilising the neurological deficit. ${ }^{1}$ The long-term survival of the patient is dependent upon the factors discussed previously; those patients with poor performance status, rapid deterioration, poor motor function and significant visceral disease have the poorest survival. Most MSCC patients have a limited life expectancy of only a few months, although a number of patients whose prognosis is favourable do survive for much longer. ${ }^{10}$ 
Radiotherapy schedules need to be individualised to take into account variability in life expectancy. Studies have shown no difference in functional outcome or overall survival between schedules, but longer treatments do provide improved control. ${ }^{11-14}$. Rades et al ${ }^{13,14}$ looked at 265 patients treated in a non-randomised prospective trial. Dutch patients received short-course treatments $(1 \times 8$ Gy or $4 \times 4$ Gy) and German patients' long-course treatments $(10 \times 3 \mathrm{~Gy}, 15 \times 2.5$ Gy or $20 \times 2$ Gy). There was no significant difference between the Dutch and German patients in motor function $37 \%$ and $39 \%$, respectively) or 1-year overall survival ( $23 \%$ and $30 \%$, respectively), but the longer treatment schedules undertaken by the German patients did improve rates of local recurrence at 1 year ( $81 \%$ and $61 \%$, respectively). Rades et al ${ }^{13,14}$ recommended that patients with favourable prognosis should be considered for long-course treatment and those with a poor prognosis be given a single 8 Gy fraction.

\section{Re-treatment}

If there is disease recurrence within the radiotherapy field, then the re-treatment options are surgery (taking into account the higher rate of wound breakdown or infection), further radiotherapy, chemotherapy or best supportive care. Relapse immediately following radiotherapy treatment may be treated by surgery in patients who are fit enough, particularly by surgery employing newer minimally invasive surgical techniques that restrict wound size. In those who relapse locally a number of months after radiotherapy, but remain well with good motor function, re-irradiation is a useful treatment option. ${ }^{15}$

\section{Rehabilitation}

Many of MSCC patients have extensive rehabilitation needs. Patients should have thromboprophylaxis with compression stockings and low-molecular-weight heparin (once a decision has been made that they are not suitable for surgery) until they are mobile. Patients should have a pressure area assessment and appropriate pressure-relieving measures must be taken to prevent pressure ulcers developing. In those for whom it is appropriate, mobilisation should commence after 48 hours with specialist input to promote optimum function. Discharge planning and rehabilitation should start from admission and should continue in the community or local hospital or hospice after discharge from the specialist centre. MSCC patients often require significant local-community-based rehabilitation and supportive care services to maximise their quality of life. Clear pathways should exist between hospital and community-based healthcare and social service teams to ensure that equipment and support for people with MSCC who are returning home are arranged in an efficient and coordinated manner. ${ }^{1}$

\section{References}

1 National Institute for Health and Care Excellence, 2008. Metastatic spinal cord compression: diagnosis and management of patients at risk of or with metastatic spinal cord compression. (CG75.) www. nice.org.uk/guidance/CG75/ [Accessed 5 August 2014].

2 Loblaw DA, Laperriere NJ, Mackillop WJ. A population-based study of malignant spinal cord compression in Ontario. Clin Oncol 2003;15:211-7.

3 Levack P, Graham J, Collie D et al. Don't wait for a sensory level listen to the symptoms: a prospective audit of delays in diagnosis of malignant cord compression. Clin Oncol 2002;14:472-80.

4 Mitera G, Loblaw A. Delays from symptom onset to treatment in malignant spinal cord compression: quantification and effect on pre-treatment neurological status. Radiother Oncol 2003;69(Suppl 1):Abstract 141.

5 Ghogawala Z, Mansfield F, Borges L. Spinal irradiation before surgical decompression adversely affects outcomes of surgery for symptomatic metastatic cord compression. Spine 2001;26:818-24.

6 Tokuhashi Y, Matsuzaki H, Oda H et al. A revised scoring system for preoperative evaluation of metastatic spine tumour prognosis. Spine 2005;30:2186-91.

7 Patchell RA, Tibbs PA, Regine WF et al. Direct decompressive surgical resection in the treatment of spinal cord compression caused by metastatic cancer: a randomised trial. Lancet 2005;366:643-8.

8 Rades D, Huttenlocher S, Dunst J et al. Matched pair analysis comparing surgery followed by radiotherapy and radiotherapy alone for metastatic spinal cord compression. J Clin Oncol 2010;28:3597-604.

9 Tancioni F, Navarria P, Pessina F et al. Early surgical experience with minimally invasive percutaneous approach for patients with metastatic epidural spinal cord compression (MESCC) and poor prognoses. Ann Surg Oncol 2012;19:294-300.

10 Chau LKK. Metastatic spinal cord compression: radiotherapy outcome. J Pain Manag 2012;5:15-31.

11 Prewett S, Ventkitaraman R. Metastatic spinal cord compression. Review of the evidence for a dose fractionation schedule. Clin Oncol 2010;22:222-30.

12 Maranzano E, Trippa F, Casale M et al. 8Gy single-dose radiotherapy is effective in metastatic spinal cord compression: results of a phase III randomized multicentre Italian trial. Radiother Oncol 2009;93:174-9.

13 Rades D, Lange M, Veninga $\mathrm{T}$ et al. Final results of a prospective study comparing the local control of short-course and long-course radiotherapy (RT) for metastatic spinal cord compression. Int J Radiat Oncol Biol Phys 2011;79:524-30.

14 Rades D, Stalpers LJ, Veninga T et al. Evaluation of five radiation schedules and prognostic factors for metastatic spinal cord compression. J Clin Oncol 2005;23:3366-75.

15 Maranzano E, Trippa F, Casale M et al. Re-irradiation of metastatic spinal cord compression: Definitive results of two randomised trials. Radiother Oncol 2011;98:234-7.

Address for correspondence: Dr P Robson, Clatterbridge Cancer Centre, Clatterbridge Hospital, Bebington, Wirral, CH63 4JY. Email: peter.robson@clatterbridgecc.nhs.uk 